\title{
47. Organizing Data Projects With Women and Minorities in Latin America
}

\author{
Eliana A. Vaca Muñoz
}

\begin{abstract}
This chapter discusses organizing data projects with women and minorities in Latin America.
\end{abstract}

Keywords: analogue dataviz, minorities, women, Latin America, data journalism education, data visualization

Chicas Poderosas (Powerful Girls) is a transnational network of journalists, designers and developers working to develop digital media projects by and for women and marginalized communities throughout Latin America. As a designer with Chicas Poderosas, my work explores the role that design can play as an agent of culture and diversity, including through interdisciplinary and participatory research to explore cultural heritage, identity, the appropriation of territories, and the recognition of women and vulnerable populations.

This chapter examines the organization of several Chicas Poderosas initiatives in Colombia and Central America. As social and cultural inequalities in Colombia widen, it is important for minorities to be heard, to share their knowledge and to be treated as equals. To this end, the Chicas Colombia team has conducted a series of collaborative workshops focusing on data journalism and associated digital media practices. In the following sections I examine two methods that we used to facilitate participation in these workshops: Analogue data collection and analogue data visualization. These approaches may be relevant to the practices and cultures of data journalism in communities and regions where connectivity, devices and technological literacies cannot be taken for granted.

Bounegru, L. and J. Gray (eds.), The Data Journalism Handbook: Towards a Critical Data Practice. Amsterdam: Amsterdam University Press, 2021 DOI 10.5117/9789462989511_CH47 


\section{Analogue Data Collection}

In May 2016, Chicas Colombia went to Villa España in Quibdó, Chocó, to work with women and teenagers belonging to the AJODENIU (Association of Displaced Youth) collective. Since 2002 this group has worked to defend the interests and rights of children displaced from Chocó, Río Sucio, Bojayá and Urabá. These regions are all difficult to access, with no Internet and few support services available. Therefore, the workshops began with analogue techniques to collect qualitative data. With this data, we worked to construct stories on issues such as forced displacement and teenage pregnancy, by recording spoken and written narratives. ${ }^{1}$

Building on these approaches, we worked with the United Nations Development Programme in Honduras in September 2018 to create a workshop with the Observatorios de Municipales de Convivencia y Seguridad Ciudadana (Municipal Observatories for Coexistence and Citizen Security). They worked with data on violent deaths of men and women and were interested in presenting data disaggregated by gender. Two of the goals were to create emotional pathways to initiate conversations with the community around these difficult topics through participatory activities, and to use limited resources to share sensitive and important data.

At these workshops the initial steps are ice-breaking activities with simple and funny questions (Figure 47.1). At the Honduras workshop there were difficulties in discussing violence with participants due to different societal norms as well as language barriers. Thus, we focused the workshop on different exploratory data-gathering activities to surface different conceptions and experiences of violence. We used drawings, pictures and photographs to create posters together. Participants could add stickers to these as a way to gather data-including on the way they envisaged themselves, on their understanding of rights and on how they had experienced different kinds of domestic violence (e.g., physical, psychological, economic) in their own lives.

\section{Analogue Data Visualization}

In an effort to better understand the issues plaguing Indigenous communities, in 2017 we planned interactive workshops with the Embera Tribe of the Vigía del Fuerte region. The workshops sought to provide a window into

1 https://chicaspoderosas.org/2016/11/22/the-pacific-counts-chicas-poderosas-quibdocolombia/ 


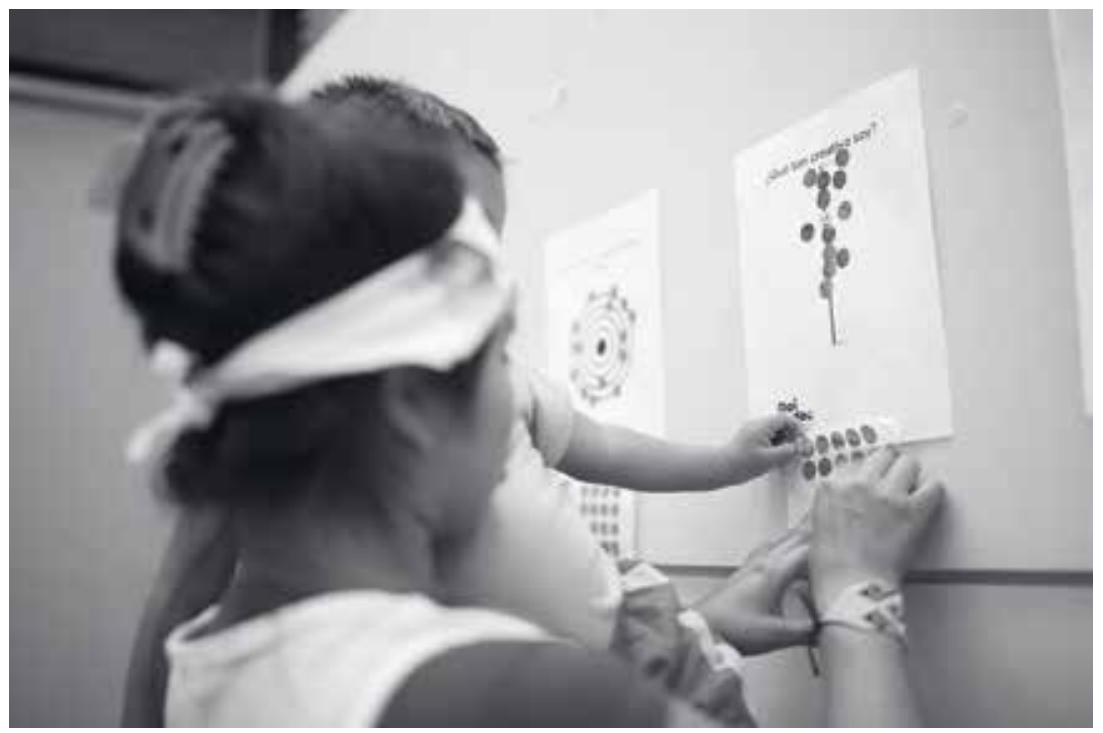

Figure 47.1. "I'm so creative?": analogue data collection activity. Source: Eliana Vaca.

their lives in spite of language barriers. Historically, interactions between the tribe and outsiders have been largely male-dominated, so we prioritized accessing the female populations in order to gauge their levels of education and facilitate discussions regarding empowerment.

In the absence of modern technologies, we explored traditional expressions of culture as a means to more meaningfully access the lives of our participants. These expressions included traditional practices such as weaving, beading and craftwork (Figure 47.2).

In September 2018 in Honduras, we ran a workshop around the question of how to "humanize" data, conducting resiliency projects with victims and populations at risk. We designed low-cost analogue data visualization workshops with empathetic design techniques using scissors, papers, stickers, post-its and balloons. These served to facilitate the sharing of sensitive information with relevant organizations to better support these communities, as well as teaching different methods that vulnerable and low-literacy populations could use to share data about their lives, experiences and issues. For example, we worked with participants to create analogue visualizations about murders and femicides by region, type and age.

In another workshop in Belize we explored different collaborative approaches to visualizing data about crime and violence. We originally set out to see how data from the Belize Violence Observatory could be used to coordinate different kinds of collective responses. While participants had 


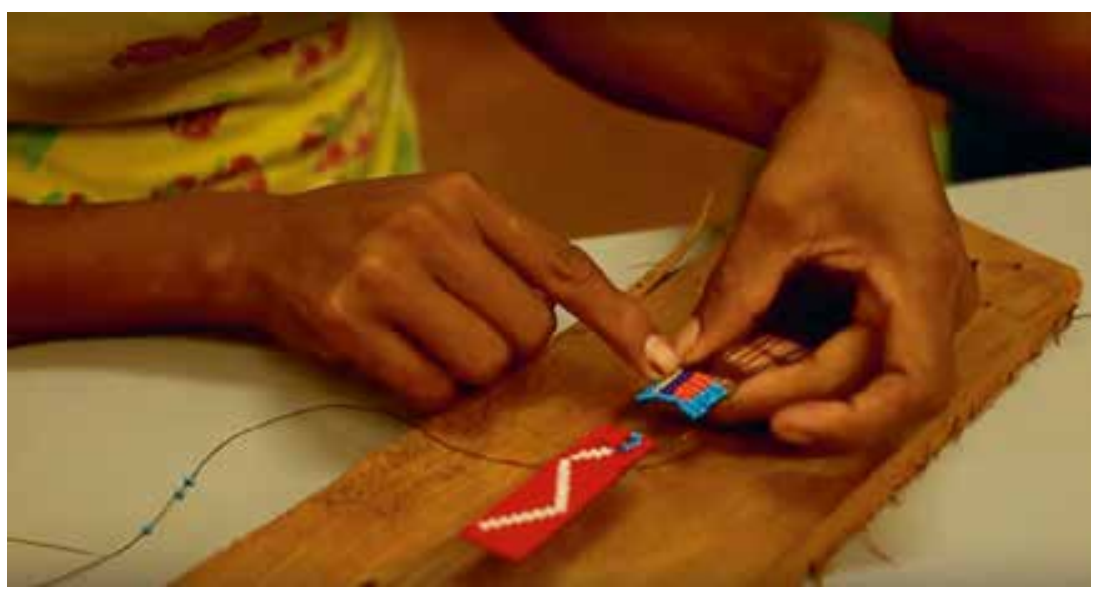

Figure 47.2. An example of analogue visualisation with beading where different colours represent different languages spoken and the amount of beads represents fluency in each. Source: Eliana Vaca.

high levels of literacy, the technological resources and connectivity were much more precarious, making it difficult to use basic online visualization tools. This raised many questions and challenges about online data visualization practices, which are often taken for granted, but which would not work in the settings we were in-again suggesting the relevance of analogue approaches to data visualization using more readily available materials.

\section{About the Author}

Eliana A. Vaca Muñoz is a designer who specializes in developing projects that involve human behaviour and anthropology methodologies to create human-centred design solutions. 
